\title{
Aplicação dos métodos FZl e zoneamento estatístico no Campo de Namorado
}

Marcella Mayara C. A. Aragão (UFCG), José Agnelo Soares* (UFCG)

Copyright 2016, SBGf - Sociedade Brasileira de Geofísica

Este texto foi preparado para a apresentação no VII Simpósio Brasileiro de Geofísica, Ouro Preto, 25 a 27 de outubro de 2016. Seu conteúdo foi revisado pelo Comitê Técnico do VII SimBGf, mas não necessariamente representa a opinião da SBGf ou de seus associados. É proibida a reprodução total ou parcial deste material para propósitos comerciais sem prévia autorização da SBGf.

\section{Abstract}

The potentiality of petroleum reservoirs depends on some parameters of the rock, as porosity and permeability. In order to the fluid be stored and produced efficiently it is necessary that these parameters are high. Techniques and tools developed to carry out the process of reservoir characterization use of majority form core data, being a challenge the large amount of data. The techniques used in this work are statistical zoning and flow zone indicator $(\mathrm{FZI})$. With the use of these techniques it is possible to get the different flow units. This work aims to apply these techniques at the Namorado petroleum field and compare the results achieved through both approaches. Permeability core data from 15 wells was used in this research. The definition of flow zones was obtained from the calculation of the variance between zones, from variances grouped within zones and, finally, from the zoning index. The reconnaissance of zones by the FZI method is based on the different proportions between the well logging curves. The techniques of statistical and FZI zoning have produced equivalent results in some wells, but divergent ones in others, with no clear indication which method offers better general performance.

\section{Introdução}

Reservatórios de hidrocarbonetos devem ser constituídos por rochas que apresentem porosidade e permeabilidade suficientemente elevadas, de modo a serem capazes de armazenar e produzir os fluidos neles contidos (MARQUES, 2011). O processo de caracterização define os principais parâmetros petrofísicos usados para prever o comportamento do fluxo de fluidos no interior da formação porosa. Esse processo utiliza, majoritariamente, dados obtidos em testemunhos de poços (MUSTAFA, 2003). Segundo Testerman (1962), entre os principais desafios para prever ou interpretar 0 comportamento dos fluidos é organizar e utilizar a grande quantidade de dados de testemunhos.

À medida que o fluxo de fluido é observado são necessárias aplicações de técnicas que determinem a posição real das zonas de fluxo do reservatório e suas propriedades. Devido à complexidade dessa tarefa, ferramentas como a estatística vêm sendo utilizadas para reconhecer as zonas de fluxo do reservatório (MUSTAFA, 2003). Outra técnica que pode ser aplicada com a finalidade de obter informações sobre as zonas de fluxo é o indicador de zona de fluxo (FZI). O FZI é uma técnica que auxilia na quantificação do fluxo de fluidos no reservatório e a partir dela é possível obter uma relação entre diferentes propriedades petrofísicas (AL-DHAFEERI et al,. 2007).

Este trabalho tem como objetivo aplicar as técnicas de zoneamento estatístico e FZI no campo de Namorado, da Bacia de Campos, e comparar os resultados.

\section{Metodologia}

Os dados utilizados para a execução deste projeto foram disponibilizados pela Agência Nacional de Petróleo (ANP), que elegeu o campo de Namorado como "campo escola" e ter parte dos seus dados livres para fins de pesquisa acadêmica.

Foram selecionados quinze poços do Campo de Namorado, NA01A, NA02, NA04, NA07, NA11A, NA12A, NA22A, NA37D, NA40, NA44, NA47D, NA48D, NA53D, RJS42 e RJS234. A preferência pelo estudo destes justifica-se com 0 fato de possuírem valores de permeabilidade distribuídos ao longo dos poços e por possuírem todas as curvas básicas de perfilagem geofísica. Após a obtenção dos dados foi realizada a etapa de organização e seleção do que seria utilizado para a aplicação das técnicas de zoneamento estatístico e o indicador de zona de fluxo (FZI).

A primeira etapa deste trabalho foi a aplicação do zoneamento estatístico. Os dados de permeabilidade de cada poço foram reunidos com o objetivo de aplicar as equações 1 a 3 . As zonas são selecionadas de modo que a variação seja minimizada no interior da zona e maximizada entre as zonas.

$$
\begin{gathered}
B=\frac{1}{L-1}\left[\sum_{i=1}^{L} m_{1}\left(\bar{k}_{i}-\bar{k} . .\right)^{2}\right] \\
=\frac{1}{L-1}\left[\sum_{i=1}^{L} \frac{\left(\sum_{j=1}^{m_{i}} k_{i j}\right)^{2}}{m_{i}}-\frac{\left.\sum_{i=1}^{L} \sum_{j=1}^{m_{i}} k_{i j}\right]}{N}\right] \\
W=\frac{1}{N-L}\left[\sum_{i=1}^{L} m_{i} \sum_{j=1}^{m_{i}}\left(k_{i j}-\bar{k}_{i}\right)^{2}\right] \\
=\frac{1}{N-L}\left[\sum_{i=1}^{L} \sum_{j=1}^{m_{i}} k_{i j}^{2}\right. \\
\left.-\sum_{i=1}^{L} \frac{\left(\sum_{j=1}^{m_{i}} k_{i j}\right)^{2}}{m_{i}}\right] \\
R=\frac{B-W}{B}
\end{gathered}
$$


As Equações 1, 2 e 3 representam a variância entre zonas, à variância agrupada dentro das zonas e o índice de zoneamento, respectivamente.

Pela Equação 3 é possível verificar a melhor divisão possível de zonas. Este índice varia entre 0 e 1. Quando o valor de $\mathrm{R}$ estiver próximo de 1 significa que as zonas apresentam uma boa homogeneidade e portanto uma boa divisão de zonas. Após a combinação de duas zonas serem obtidas, os dados foram divididos em todas as possíveis combinações para três e quatro zonas, utilizando o mesmo critério utilizado na classificação em duas zonas.

Os dados utilizados para aplicar a técnica FZI foram obtidos nos perfis geofísicos dos poços. As equações 4 a 9 foram então utilizadas para a obtenção das zonas de fluxo.

$$
\begin{aligned}
& G R_{T r}=4.7860 * 10^{-3} * G R^{2}-1.732 * 10^{-1} * G R \\
& +1.0614 \\
& N P H I_{T r}=-8.1102 * N P H I^{2}+9.6676 * 10^{-1} * N P H I \\
& +1.7170 * 10^{-1} \\
& R H O B_{T r}=7.1926 * R H O B^{2}-3.6727 * 10^{1} * R H O B \\
& +4.5873 * 10^{1} \\
& I L D_{T r}=-1.6859 * 10^{-4} * I L D^{2}-3.8016 * 10^{-2} * I L D \\
& +4.3712 * 10^{-1} \\
& S O M A=G R_{T r}+N P H I_{T r}+I L D_{T r}+R H O B_{T r}(8) \\
& F Z I=4.4306 * 10^{-1} * S O M A^{2}+6.08575 * 10^{-1} * S O M A \\
& +3.8229 * 10^{-1}
\end{aligned}
$$

\section{Resultados}

As Tabelas 1 a 3 apresentam as profundidades indicadas pelos métodos de zoneamento estatístico e o indicador da zona de fluxo (FZI). As profundidades apresentadas por cada um dos métodos indicam a localização ideal das interfaces entre as zonas de fluxo.

Através do método de zoneamento estatístico as zonas foram divididas a partir do cálculo do índice de zoneamento. Para todos os poços foram calculadas as Equações 1,2 e 3. Quando o índice foi obtido e seu valor estava entre 0,98 e 1,00 era verificado que aquela era a melhor divisão de zonas. Para índices inferiores o método foi aplicado novamente até que a diferença dos índices fosse igual ou inferior a 0,06 ou para $R$ próximo de 1.

Enquanto que para o método $\mathrm{FZI}$ verificou-se o mesmo intervalo de profundidade usada no zoneamento estatístico e observou-se qual o maior valor do FZI, esta análise foi feita para os diferentes intervalos de profundidade nas diferentes divisões de zonas.
Tabela 1: Resultados obtidos nas técnicas de zoneamento estatístico e FZI para duas zonas.

\begin{tabular}{|c|c|c|c|}
\hline Poços & $\begin{array}{c}\text { Profundidade da } \\
\text { interface entre } \\
\text { zonas - } \\
\text { Zoneamento }(\mathrm{m})\end{array}$ & $\begin{array}{c}\text { Profundidade } \\
\text { da interface } \\
\text { entre zonas - } \\
\text { FZI }(\mathrm{m})\end{array}$ & $\mathrm{R}$ \\
\hline NA01 & 3309,0 & 3309,8 & 0,98 \\
\hline NA02 & 3048,3 & 3042,6 & 0,98 \\
\hline NA04 & 3046,2 & 3039,8 & 0,98 \\
\hline NA07 & 3089,0 & 3091,2 & 0,91 \\
\hline NA11 & 3139,2 & 3076,4 & 0,83 \\
\hline NA12 & 3053,9 & 3079,1 & 0,79 \\
\hline NA22 & 3190,2 & 3190,7 & 1,00 \\
\hline NA37 & 3221,5 & 3221,9 & 0,42 \\
\hline NA40 & 3152,0 & 3148,9 & 0,89 \\
\hline NA44 & 3308,0 & 3306,4 & 0,72 \\
\hline NA47 & 3258,6 & 3258,4 & 0,64 \\
\hline NA48 & 3188,7 & 3191,9 & 0,76 \\
\hline NA53 & 3224,0 & 3225,0 & 0,89 \\
\hline RJS42 & 3155,5 & 3144,6 & 0,88 \\
\hline RJS23 & 3231,2 & 3233,5 & 0,26 \\
\hline 4 & 305 & & \\
\hline
\end{tabular}

Tabela 2: Resultados obtidos nas técnicas de zoneamento estatístico e FZI para três zonas.

\begin{tabular}{|c|c|c|c|}
\hline Poços & $\begin{array}{c}\text { Profundidade da } \\
\text { interface entre } \\
\text { zonas - } \\
\text { Zoneamento }(\mathrm{m})\end{array}$ & $\begin{array}{c}\text { Profundidade } \\
\text { da interface } \\
\text { entre zonas - } \\
\text { FZI }(\mathrm{m})\end{array}$ & $\mathrm{R}$ \\
\hline NA07 & 3088,6 & 3088,7 & 0,97 \\
\hline NA11 & 3123,9 & 3076,3 & 0,93 \\
\hline NA22 & 3184,3 & 3190.2 & 0,98 \\
\hline NA40 & 3156,2 & 3156,8 & 0,98 \\
\hline NA44 & 3295,6 & 3306,3 & 0,88 \\
\hline NA53 & 3226,4 & 3224,9 & 0,83 \\
\hline RJS42 & 3140,6 & 3144,3 & 0,85 \\
\hline RJS23 & 3230,4 & 3225,5 & 0,71 \\
4 & & & \\
\hline
\end{tabular}

Tabela 3: Resultados obtidos nas técnicas de zoneamento estatístico e FZI para quatro zonas.

\begin{tabular}{|c|c|c|c|}
\hline Poços & $\begin{array}{c}\text { Profundidade da } \\
\text { interface entre } \\
\text { zonas - } \\
\text { Zoneamento }(\mathrm{m})\end{array}$ & $\begin{array}{c}\text { Profundidade } \\
\text { da interface } \\
\text { entre zonas - } \\
\text { FZI }(\mathrm{m})\end{array}$ & $\mathrm{R}$ \\
\hline NA11 & 3137,2 & 3134,8 & 0,93 \\
\hline NA22 & 3187,7 & 3190,2 & 0,99 \\
\hline RJ234 & 3231,2 & 3231,5 & 1,00 \\
\hline
\end{tabular}




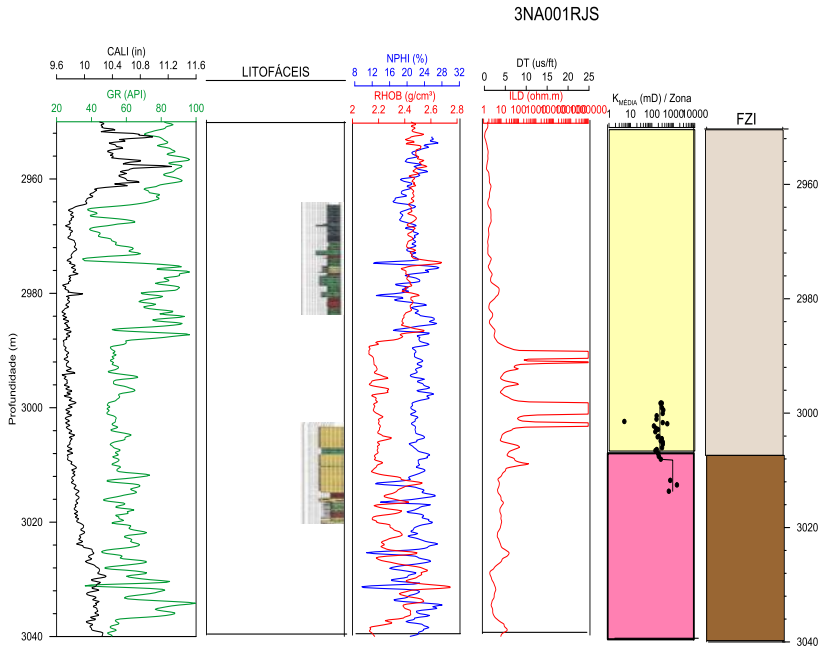

Figura 1: Perfil do poço NA01.

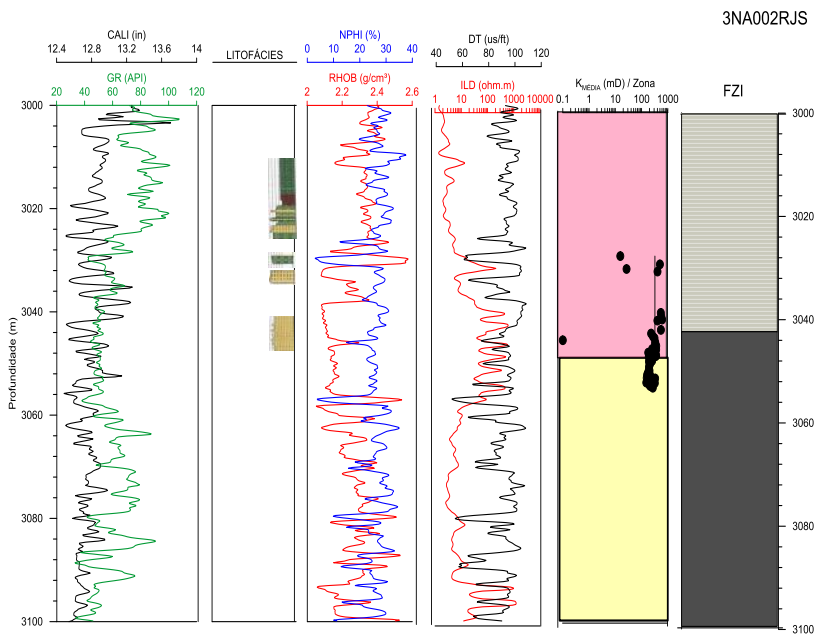

Figura 2: Perfil do poço NA02.

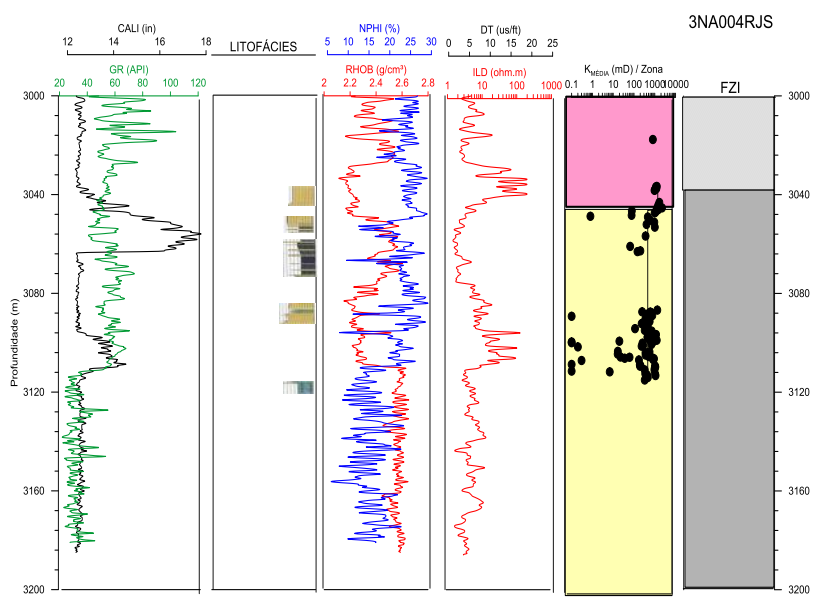

Figura 3: Perfil do poço NA04.

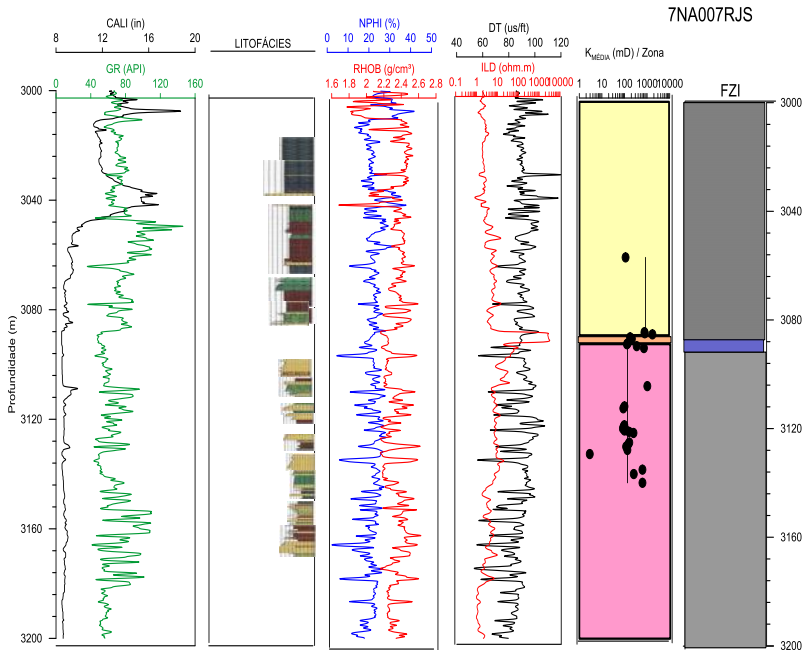

Figura 4: Perfil do poço NA07.

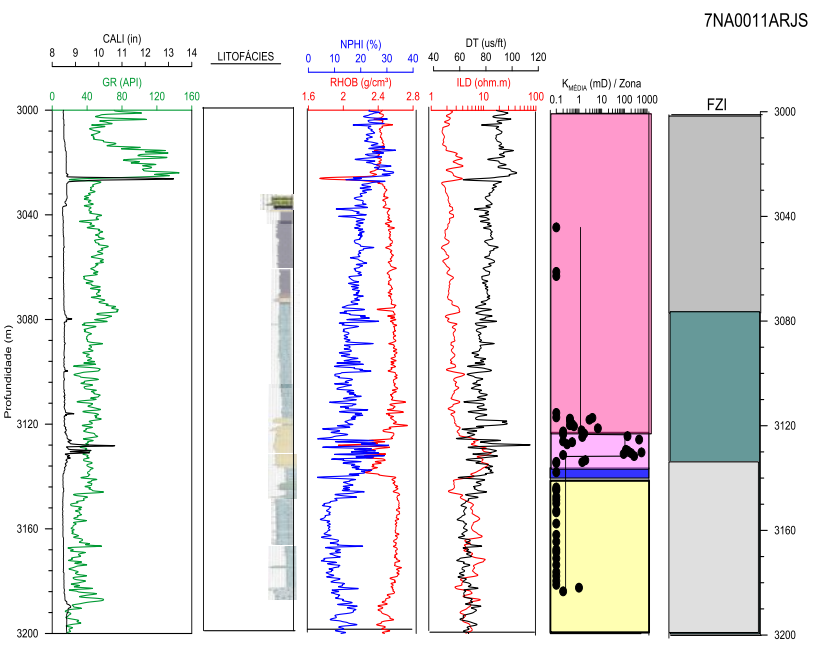

Figura 5: Perfil do poço NA11.

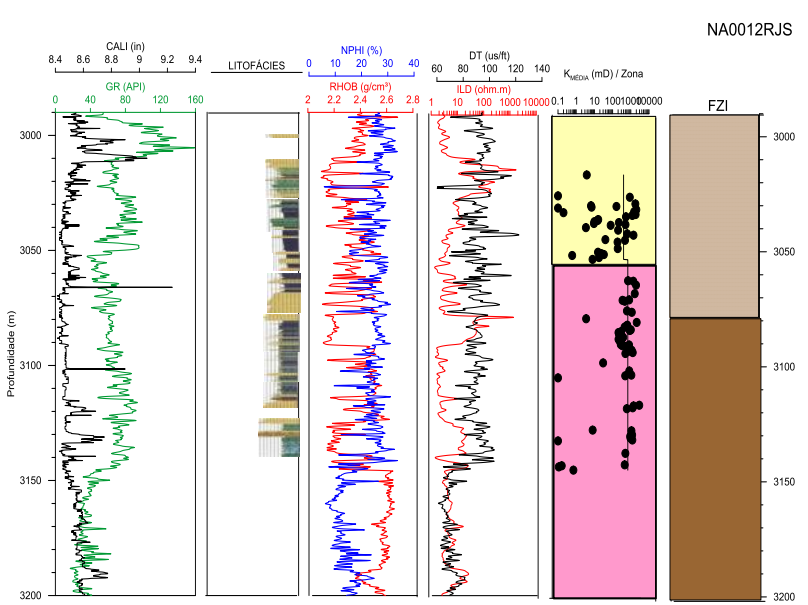

Figura 6: Perfil do poço NA12. 


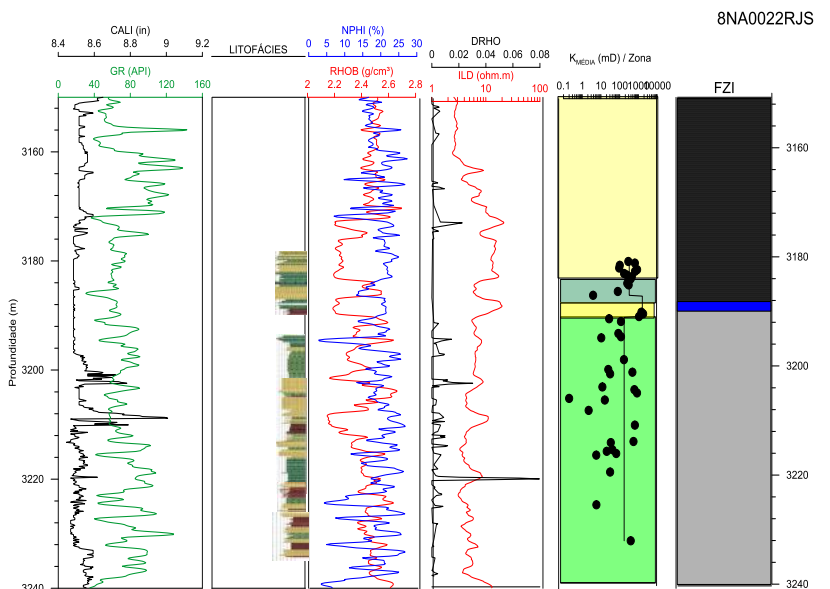

Figura 7: Perfil do poço NA22.

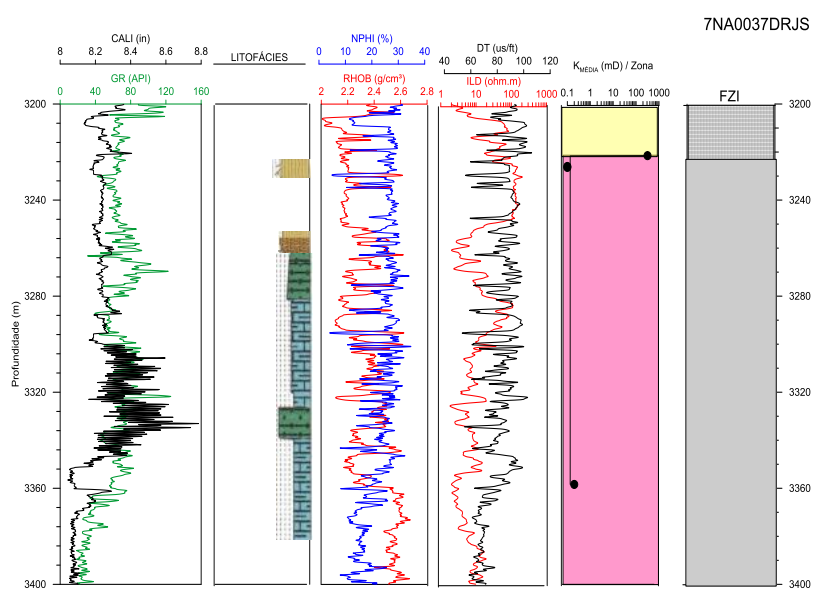

Figura 8: Perfil do poço NA37.

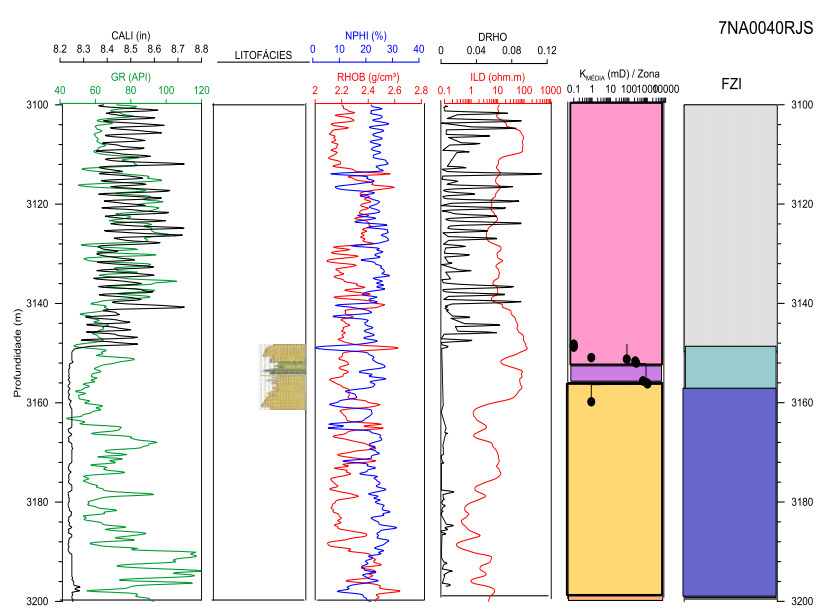

Figura 9: Perfil do poço NA40.

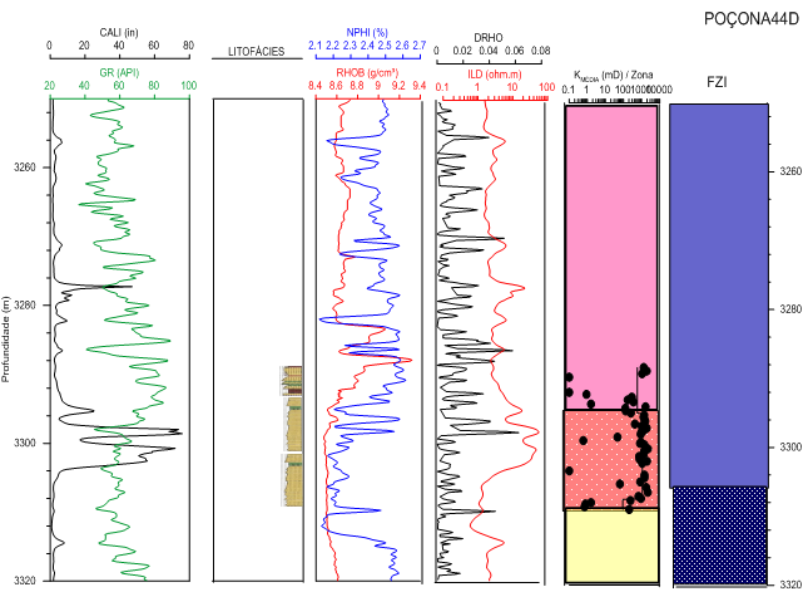

Figura 10: Perfil do poço NA44.

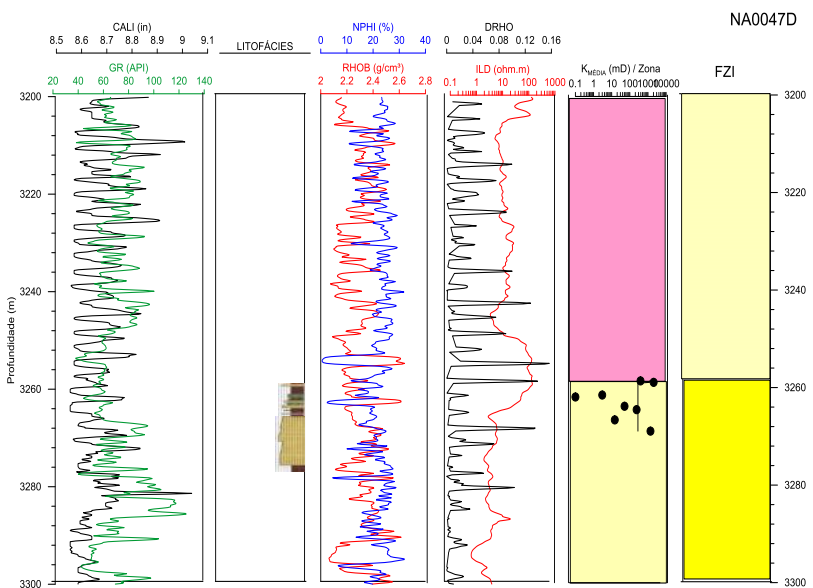

Figura 11: Perfil do poço NA47.

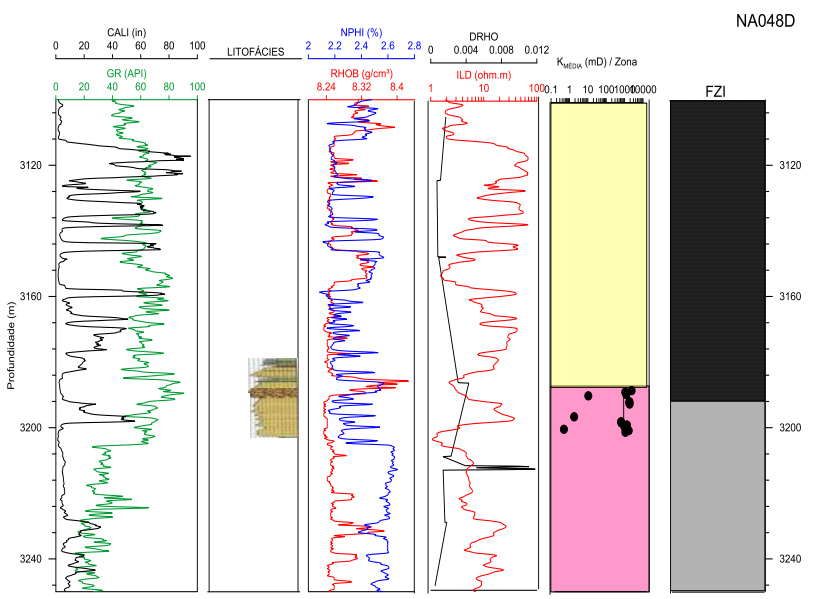

Figura 12: Perfil do poço NA48. 


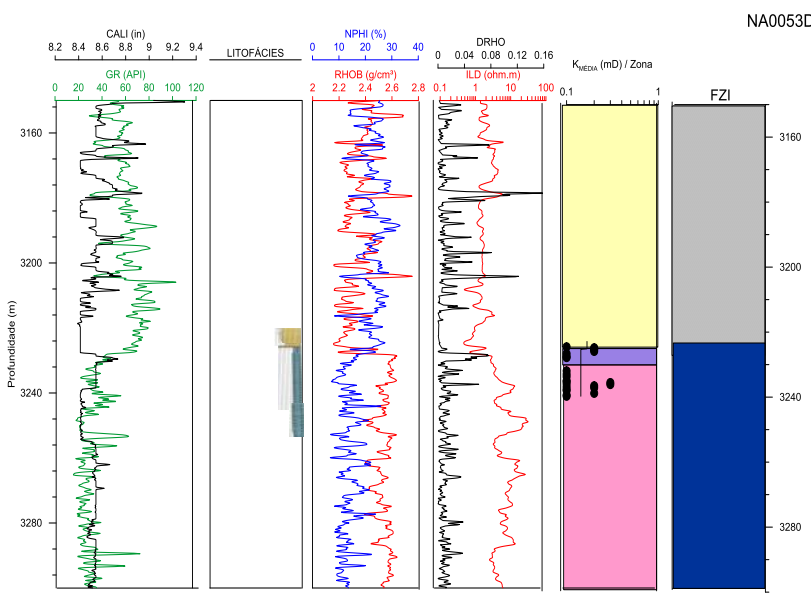

Figura 13: Perfil do poço NA53.

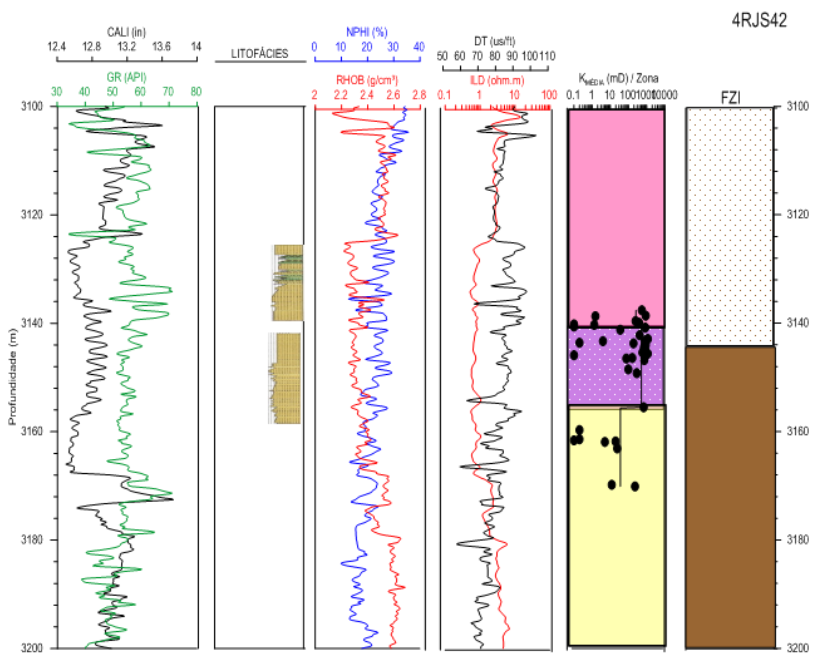

Figura 14: Perfil do poço RJS42.

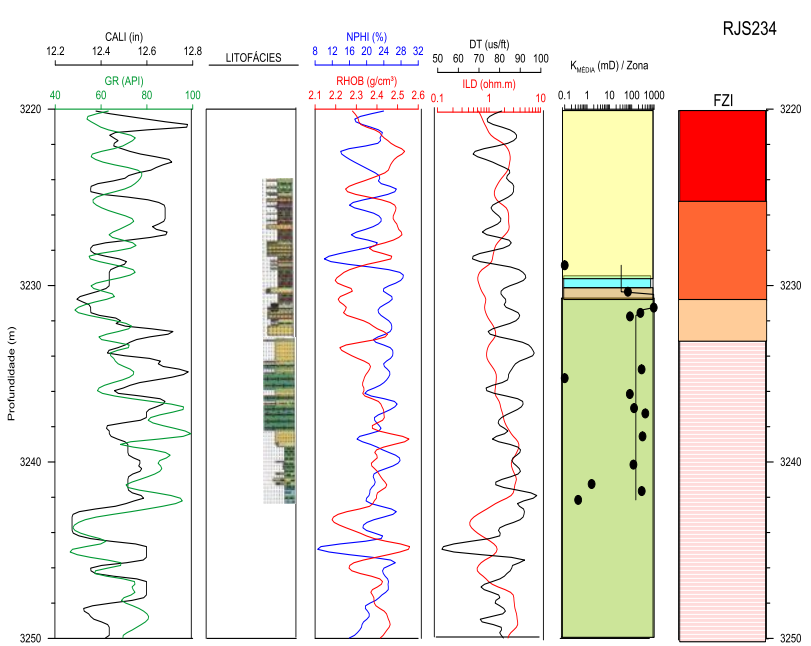

Figura 15: Perfil do poço RJS234.

\section{Discussão e Conclusões}

A Figura 1 apresenta o perfil do poço NA01. Observa-se que os dois métodos reconhecem duas zonas cuja interface coincide.

As Figuras 2 e 3 mostram as zonas obtidas a partir das técnicas de zoneamento estatístico e FZI para os poços NA02 e NA04. Embora ambos os métodos indiquem duas zonas há uma defasagem de cerca de 6 metros na profundidade da interface.

A Figura 4 apresenta o perfil do poço NA07. Verifica-se que os dois métodos indicaram a presença de três zonas com profundidades de interfaces semelhantes.

A Figura 5 mostra o perfil do poço NA11. Os dois métodos apontaram a mesma quantidade de zonas. Porém, observa-se a partir das curvas que o zoneamento estatístico apresentou melhores resultados.

A Figura 6 mostra o perfil do poço NA12. Os métodos verificaram a presença de duas zonas, porém observouse uma diferença de 25 metros nas profundidades das interfaces. A partir da análise do perfil e as respostas geradas pelos métodos verifica-se que o $\mathrm{FZI}$ parece indicar uma melhor resposta.

A Figura 7 apresenta o perfil do poço NA22. Através do zoneamento estatístico foi detectada a presença de quatro zonas, enquanto que pelo $\mathrm{FZI}$ identificou-se três zonas. Com relação às profundidades das interfaces existiu uma grande semelhança entre elas.

As Figuras 8, 11 e 12 mostram os perfis dos poços NA37, NA47 e NA48. Percebe-se que os dois métodos indicam a divisão de duas zonas, cuja interface coincide.

A Figura 9 expõe o perfil do poço NA40. Apesar de ambos os métodos indicarem o mesmo número de zonas (três), observa-se a partir dos perfis que o FZI pareceu ter resultados mais satisfatórios em comparação com 0 zoneamento estatístico.

O perfil do poço NA44 é apresentado na Figura 10. As técnicas identificaram diferentes números de zonas, foi possível observar através da curva de resistividade (ILD) que a divisão através do FZI parece um pouco melhor que a divisão pelo zoneamento.

A Figura 13 mostra o perfil do poço NA53. Os métodos indicaram diferentes números de zonas, porém as interfaces foram semelhantes. Verificou-se a partir do perfil que o $\mathrm{FZI}$ gerou resultados melhores.

A Figura 14 e 15 mostram os perfis dos poços RJS42 e RJS234, respectivamente. Na Figura 14 houve uma diferença no número de zonas, mas não há uma indicação clara de um método ter sido melhor que o outro. Enquanto que na Figura 15 ambos os métodos indicam quatro zonas, mas as interfaces parecem melhor situadas pelo método de zoneamento estatístico.

Com o objetivo de realizar uma comparação entre o desempenho dos métodos zoneamento estatística e FZI conclui-se que em alguns casos ambos os métodos apontaram para o mesmo número de zonas. Mas não é possível inferir qual método apresenta um melhor 
resultado já que em alguns casos um foi melhor que outro.

\section{Referências}

Al-Dhafeeri, A. M., Nasr-El-Din, H.A., 2007. Characteristics of high permeability zones using core Analysis and production logging data. Journal of Petroleum Science and Engg. January, 13-25.

Marques, E. A. R., 2011. Caracterização de Reservatórios Petrolíferos. Dissertação (Engenharia Geológica e de Minas), Universidade Técnica de Lisboa.

Mustafa, R., 2003. Application of Statistical Methods for "Flow Unit" Identification and Characterization of a Reservoir Using Well Log and Core Data. Thesis Submitted to the College of Engineering and Mineral Resources at West Virginia University in partial fulfillment of the requirements for the degree of Master of Science in Petroleum and Natural Gas Engineering.

Testerman, J. D., 1962. "A Statistical Reservoir-Zonation Technique." Soc. Petrol. Engr. JPT 